\title{
Höhere Integrierbarkeit und Regularität für eine Klasse \\ freier Randwertprobleme
}

\author{
M. Fochs
}

Es' werden Funktionen $u: B^{+}:=\left\{x \in \mathbf{R}^{n}:|x| \leqq 1, x^{n} \geqq 0\right\} \rightarrow \bar{G}$ der Sobolev-Klasse $H^{1, p}$ betrachtet, die das Variationsintegral $\int|D v|^{p} d x$ für ein $p, 2 \leqq p \leqq n$, unter der Nebenbedingung $\operatorname{Im}(v) \subset \bar{G}$ und der freien Randbedingung $v(x) \in \Sigma$ für $x \in \partial B^{+}$mit $x^{n}=0$ lokal minimieren. Hierbei ist $\bar{G}$ der Abschluß eines glatten Gebietes $G \subset \mathbf{R}^{N}$ und $\Sigma$ eine kompakte Untermannigfaltigkeit in $\bar{G}$. Es wird höhere Integrierbarkeit bis zum freien Rand gezeigt; in konform-invarianten Fall sind die Minimanten Hölder-stetig bis auf $\partial B^{+} \cap\left\{x: x^{n}=0\right\}$.

Рассматриваются фуниции $u: B^{+}:=\left\{x \in \mathbf{R}^{n}:|x| \leqq 1, x^{n} \geqq 0\right\} \rightarrow \dot{\bar{G}}$ класса Соболева $H^{1 . p}$, которые ілокально минимизируют вариационный интеграл $\int_{B^{+}}^{-}|D v|^{p} d x$ для некоторого $p, 2 \leqq p \leqq n$, под дополнительиым условием $\operatorname{Im}(v) \subset \bar{G}$ и под свобопным граничиым условием $v(x) \in \Sigma$ для $x \in \partial B^{+}$с $x^{n}=0$. Злесь $\bar{G}-$ замыкание гладкой области $G \subset \mathbf{R}^{N}$ и. $\Sigma$ - компактиое попмногообразие в $\bar{G}$. Доказывается высшая интегрируемость доо свободиой, границы; в конформно-инвариантном случае миниманты непрерывны по Гельдеру до $\partial B^{+} \cap\left\{x: x^{n}=0\right\}$.

Functions $u: B^{+}:=\left\{x \in \mathbf{R}^{n}:|x| \leqq 1, x^{n} \geqq 0\right\} \rightarrow \bar{G}$ of Sobolev class $H^{1, p}$ are conisdered which locally minimize the variational integral $\int_{B^{+}}|D v|^{p} d x$ for some $p, 2 \leqq p \leqq n$, under the side condition $\operatorname{Im}(v) \subset \bar{G}$ and the free boundary condition $v(x) \in \Sigma$ for $x \in \partial B^{+}$such that $x^{n}=0$. Here $\bar{C}$ is the closure of a smooth domain $G \subset \mathbf{R}^{N}$ and $\Sigma$ denotes a compact submanifold contained in $\bar{G}$. Higher integrability up to the free boundary is proved; in the conformally invariant case minimants are Hölder. continuous up to $\partial B^{+} \cap\left\{x: x^{n}=0\right\}$.

\section{Einführung}

Gegenstand der Arbeit ist die Untersuchung des Verhaltens vektorwertiger Funktionen, die ein freies Randwertproblem mit zusät\%licher Hindernisbedingung lösen. Genauer"betrachten wir für Exponenten $p \in[2, n]$ Abbildungen

$$
u: B_{1}^{+}(0):=\left\{z \in \mathbf{R}^{n}: z^{n}>0,|z|<1\right\} \rightarrow \bar{G}
$$

der Sobolev-Klasse $H^{1, p}$ und ein Funktional der Form

$$
E(u):=\int_{B_{1}^{+}(0)}|D u|^{p} d x
$$

wobei $u$ diese $p$-Energie lokal minimiert unter allen Funktionen $v: B_{1}{ }^{+}(0) \rightarrow G$, welche die freie Randbedingung $v(x) \in S$ für $x \in \partial \dot{B}_{1}{ }^{+}(0) \cap \mathbf{R}^{n-1}$ erfüllen. Hierbei ist $G$ ein glatt berandetes Gebiet in einer Riemannschen Mannigfaltigkeit $Y$, und $S$ bezeichnet eine Untermannigfaltigkeit von $Y$ mit $S \subset \bar{G}$. Da alle zur Konkurrenz zugelasseñen Funktionen $v$ ihr Bild im Abschluß von $G$ haben, sind wir gleichzeitig mit einem Hindernisproblem konfrontiert. Die. zugehörige (partielle) Regularitäts- 
theorie bei Dirichlet-Randdaten und im Spezialfall $p=2$ wurde von DuzaAr und dem Autor in [D], [D, F 1], [D, F 2], [F 1 -5] ausführlich beschrieben. GoRnIK [Go] zeigte. innere Regularität im konform invarianten Fall $p=n$; und in. [F6] wurde vom Autor folgende Abschätrung für die innere Singularitätenmenge Sing beim $\bar{p}$-harmonischen Hindernisproblem bewiesen:

$$
\begin{aligned}
& \mathbf{H}-\operatorname{dim}(\text { Sing }) \leqq n-[p]-1, \text { falls } p<n-1, \\
& \text { Sing ist diskret für } p \geqq n-1 .
\end{aligned}
$$

Dagegen ist die Regularitätsfrage am freien Rand bisher wenig untersucht. Am besten bekannt ist das Randverhalten von Minimalflächen und Flächen vorgeschriebener mittlerer Krümmung wie es von GrüTER, HILd EBRaN DT, JÄGER, Jost, NITSChE und anderen beschrieben wird, man vergleiche hierzu etwa $[G, H, N$ ] oder [J1] und die dort zitierten Arbeitén. Allerdings sind die benut»ten Argumente zweidimen: sionaler Natur und erlauben kaum eine Ausdehnung auf allgemeinere Situationen.

Das freie Randwertproblem für harmonische Abbildungen (d. h. $p=2, G=Y$ ) wird erstmals von BaLDES [B] angegriffen mit folgendem Ergebnis: ist die ,Stiitzfläche" $S$ total geodätisch und hat die betrachtete Minimalstelle ihr Bild in einer sogenannten regulärèn Kugel, so gilt ein Randregularitätssatz. In [G, J] wird dieses Resultat verallgemeinert, überdies wird mit einem Beispiel belegt, daß ohne Einschränkungen an die Geometrie singuläre Randpunkte auftreten können.

Das Ziel dieser Arbeit besteht darin, dịe Grundzüge einer partiellen Reguläritätstheorie am freien Rand zu entwickeln. Ausgangspunkt ist die in [F6] beschriebene Technik der partiellen höheren Integrierbarkeit, die zum Beweis des folgenden inneren Regularitätssatzes für das $p$-harmonische Hindernisproblem führte:

Es gibt ein $\varepsilon$ abhängig von geometrischen Daten mit der Eigenschaft, daß aus $r^{p-n} \int_{B_{r}(x)}|D u|^{p}<\varepsilon$

für eine Kugel $B_{r}(x)$ die Stetigkeit der,Minimalstelle u lokal bei $x$ folgt.

Die Kleinheit der, skalierten $p$-Energie liefert nämlich mit der Monotonieformel und dem Fortsetzungssatz (vgl. Abschnitt 2) einen Ersatz für die Caccioppoli-Ungleichung:

$$
\underset{B_{l}(y)}{f^{\prime}|D u|^{p} d z \leqq \frac{1}{2}} \underset{B_{1}(y)}{f}|D u|^{p} d z+\frac{C}{t^{p}} \cdot f_{B_{2}(y)}\left|u-(u)_{2 t}\right|^{p} d z
$$

für Radien $t \leqq r / 2$ und Punkte $y$ nahe $x$, also $D u \in L^{p+\delta}$ lokal bei $x$ für ein geeignetes $\delta>0$. Höhere Integrierbarkeit kombiniert mit dem Eulersystem für $u$ impliziert dann $x \in \operatorname{Reg}(u)$, für die Einzelheiten sei auf [F6], Kapitel 5, verwiesen.

Wir zeigen in dieser Arbeit (vgl. Satz 2), da B partielle höhere Integrierbarkeit auch am freien Rand gilt: Löst $u$ das eingangs beschriebene Problem, so impliziert die Kleinheit von $f_{B_{1}+(0)}|D u|^{p} d x$ höhere Integrierbarkeit von $D u$ auf $B_{1 / 2}^{+}(0)$. Im konforn invarianten Fall $p=n$ ergibt sich als Korollar die Hölder-Stetigkeit von $u$ am freien Rand. Die Beweismethode benutzt eine Version der Monotonieformel bis zum freien Rand und den aus [F6] entnommenen Fortsetzungssatz. Es sei noch" 'bemerkt, cla $B$ höhere Integrierbarkeit am freien Rand mit den Argumenten aus [F6] ' (Beweis von Theorem $A^{\prime}$ ) sofort partielle Randregularität liefert, wenn man das für Minimalstellen auf $B_{1}{ }^{+}(0)$ gültige Eulersystem durch Spiegeln auf die gainze Kugel fortsetzen kann. 


\section{1.' Bezeichnungen und Ergebnišse}

Es sei $Y$ eine Riemannsche Mannigfaltigkeit, die wir uns in den Euklidischen Raum. $\mathbf{R}^{N}$ eingebettet denkèn. In $Y$ sei ein beschränktes, glatt berandetes Gebiet $G$ vorgegeben mit $\bar{G} \subset Y$, und $S$ bezeichne eine kompakte Untermannigfaltigkeit von $Y$. Es gelte $S \subset \bar{G}$. Für $n \geqq 2$ seien

$$
\mathbf{R}_{+}{ }^{n}:=\left\{x \in \mathbf{R}^{n}: x^{n}>0\right\}, \quad T:=\mathbf{R}^{n-1}=\mathbf{R}^{n-1} \times\{\dot{0}\} \subset \mathbf{R}^{n} .
$$

Ist $B$ eine Kugel in $\mathbf{R}^{n}$, so steht $B^{+}$für $B \cap \mathbf{R}_{+}{ }^{n}$. Wir betrachten für $p \in[2, n]$ folgende Funktionenklasse

$$
\begin{aligned}
\dot{\Sigma}:= & \left\{u \in H_{1 \mathrm{oc}}^{1, p}\left(\mathbf{R}_{+}{ }^{n}, Y\right): u \in H^{1, p}\left(B_{r}+(0), Y\right) \text { für alle } \dot{r}>0 \text { mit } u(x) \in \bar{G}\right. \\
& \text { auf } \left.\mathbf{R}_{+}{ }^{n} \text { und } u(z) \in S \text { auf } T\right\} .
\end{aligned}
$$

Funktionen der Klasse $\Sigma$ respektieren also das Hindernis $\dot{\bar{G}}$ und bilden die Hyperebene $T$ auf die Untermannigfaltigkeit $S$ ab. Schließlich sei $f: \mathbf{R}^{n} \times \mathbf{R}^{N} \times \mathbf{R}^{n N} \rightarrow[0, \infty)$ eine Caratheodory-Funktion, die mit Konstanten $\lambda, \Lambda>0$ folgende Ungleichung erfüllt:

$$
\lambda|Q|^{p} \leqq f(x, y, Q) \leqq \Lambda|Q|^{p}, \quad \cdot(x, y, Q) \in \mathbf{R}^{n} \times \mathbf{R}^{N} \times \mathbf{R}^{n N}
$$

Für $D \subset \approx \mathbf{R}_{+}^{\prime} \cup T$ und $u \in \Sigma$ seien

$$
F(u, D):=\int_{D} f(\cdot, u, D u) d x, \quad E(u, D):=\int_{D}|D u|^{p} d x
$$

Definition: $u \in \sum$ heißt lokal $F$-.(oder $E$-) minimal, falls

$$
F\left(u, B_{r}{ }^{+}(0)\right) \leqq F\left(v, B_{r}{ }^{+}(0)\right) \quad\left(E\left(u, B_{r}{ }^{+}(0)\right) \leqq E\left(v, B_{r}{ }^{+}(0)\right)\right)
$$

gilt für alle Funktionen $v \in \Sigma$ mit $u=v$ außerhalb einer Halbkugel $B_{r}^{+}(0)$.

Wir formulieren jetzt unsere Ergebnisse.

Satz 1 (Konform invarianter Fall $p=n$ ): $E s$ sei $p=n$ und $u \in \Sigma$ lokal F-minimal. Dann, gibt ès einen Exponenten $q>n$ mil $D u \in L^{q}\left(B_{r}^{+}(0)\right)$ für alle Radien $r$, insbesondere ist u Hölder-stetig bis zum freien Rand $T$.

Satz 2 (Partielle höhere Integrierbarkeit): Das Gebiet $G$ sei so beschaffen, daß die Nächste-Punkt-Projektion $\bar{G} \rightarrow S$ wohldefiniert und glatt ist. $u \in \Sigma$ sei lokal $E$-minimal. Dann gibt es $\varepsilon>0$ und $q>p$ abhängig von geometrischen Daten mit folgender Eigenschaft:

Ist $E\left(u, B_{1}^{+}(0)\right)<\varepsilon^{p}$, so folgt $D u \in L^{q}\left(B_{1 / 2}^{+}(0)\right)$, und für Kugeln $B_{r}(x), x \in B_{1 / 2}^{+}(0)$, $r \leqq 1 / 20$, gilt die umgekehrte Hölder-Ungleichung

$$
\left(\underset{B_{r}+(x)}{f}|D u|^{q} d z\right)^{1 / q} \leqq C\left(\underset{B_{10 r}^{+}(x)}{f}|D u|^{p} d z\right)^{1 / p}
$$

Bemerkuñgen: 1. Die Existenz einer glatten, Nächsten-Punkt-Projektion $\zeta$ : $\bar{G} \rightarrow S \subset \bar{G}$ folgt_zum Beispiel dann, wenn $G$ eine kleine Tubenumgebung von $S$ ist. 2. Statt $E$ kann man in Satz 2 allgemeiner zerfallende Funktionale vom Typ

$$
\int A(\cdot, u, D u)^{p / 2} d x \quad \text { mit } \quad A(x, y, Q)=a_{\alpha \beta}(x) B^{i j}(x, y) Q_{\alpha}^{i} Q_{\beta}^{j}
$$

zulassen. 


\section{Vorbereitende Hilfssätze}

Von entscheidender Bedeutung ist der folgende

Fortsetzungssatz: Män findet Konstanten $\gamma=\gamma(p) \in(0,1]$ und $\varepsilon_{1}, \delta, \dot{\varkappa}, \tilde{x}$ und $K>0$ abhängig von $p$, den. Dimensionen und der Geometrie wie folgt:

Sind $\varphi \in H^{1 . p}\left(S_{r}^{n-1}, \bar{G}\right)$ und $a \in \mathbf{R}^{N}$ vorgegeben mit

$$
\left.E\left(\varphi, S_{r}^{n-1}\right) \cdot\left(W\left(\dot{\varphi}, S_{r}^{n-1}\right)\right)\right)_{\rangle} \leqq \varepsilon^{\times} \delta^{\gamma+1} r^{(1+\gamma)(n-1)-p}
$$

für ein $\dot{0}<\dot{\varepsilon}<\varepsilon_{1}$, so findet man eine Fortsetzung $\Phi \in H^{1, p}\left(B_{r}{ }^{n}, \bar{G}\right)$ von $\varphi$ mit der Energieabschätzung

$$
E\left(\Phi, B_{r}{ }^{n}\right) \leqq K\left\{\varepsilon r E\left(\varphi, S_{r}^{n-1}\right)+r^{1-p_{\varepsilon}-\bar{x}} W\left(\varphi, S_{r}^{n-1}\right)\right\}
$$

Hierbei wurde gesetzt $E(\psi, \cdot):=\int|D \psi|^{p}, W(\psi, \cdot):=\int|\psi-a|^{p}$, wobei die Integration über den Définitionsbereich von $\psi$ auszujühren ist.

Für den Beweis verweisen wir auf [F6], Kapitel 4; der Spezialfall $p=2$ wird in $[\mathrm{D}, \mathrm{F} 2]$ diskutiert

Der folgende Hilfssatz beschreibt das Verhalten der skalierten $p$-Energie als Funktion des Radius:

Monotonieformel: Sei $u \in \Sigma$ lokal E-minimal, $U$ bezeichne die gerade -Fortsetzung von u auf $\mathbf{R}^{n}$. Dann gilt mit einer geeigneten Konstanten $C$

$$
e\left(U, B_{t}(x)\right):=t^{p-n} \int_{B_{t}(x)}|D \dot{U}|^{p} d z^{\prime} \leq C e\left(U, B_{s}(x)\right)
$$

für alle $t \leqq$ 's und $x \in \mathbf{R}^{n}$.

Beweis: Sei $\ddot{x} \in \mathbf{R}_{+}{ }^{n}$. Fiir $s<x^{n}$ gilt die Ungleichung nach [F, H], Lemma 8.2. Im Fall von Randpunkten $x=\left(x^{\prime}, 0\right)$ hat man entsprechend .

$$
e\left(u, B_{t}{ }^{+}\left(x^{\prime}\right)\right) \leqq C e\left(u, B_{s}{ }^{+}\left(x^{\prime}\right)\right),
$$

wie die folgende Überlegung zeigt: Sei $S_{r}^{+}:=\partial B_{r}^{+}\left(x^{\prime}\right)-T$. Nach [Mo], Theorem 3.6.1(c), ist $\left.u\right|_{S_{r}+} \in H^{1, p}\left(S_{r}^{+}, \bar{G}\right)$ für fast alle Radien $r$. Sei ohne Einschränkung $x^{\prime}=0 . v(z):=u(r z /|z|), z \in B_{r}{ }^{+}\left(x^{\prime}\right)$, liegt dann in $H^{1, p}\left(B_{r}\left(x^{\prime}\right), \bar{G}\right)$. und die Spur von $v$ auf $T$ hat Bild in $S$. Mithin ist $v$ zulässige Vergleichsfunktion, und die Minimalität von $u$ führt auf (1). Durch Kombination der beiden Fälle erhält man das allgemeine Resultat. Seien dazu $x$ mit $x^{n}>0$ und $t<s$ fixiert.

Fall $1 \vdots t \leqq x^{n}$. Für $s \leqq x^{n}$ ist nichts zu zeigen, wir betrachten deshalb den

Unterfall a : $x^{n} \leqq s \leqq 2 x^{n}$. Es ist

$$
e\left(U, B_{i}(x)\right) \leqq C \epsilon\left(U, B_{x^{n}}(x)\right) \leqq C e\left(U, B_{s}(x)\right),
$$

wobei wir hier und nachfolgend mit dem Symbol $C$ alle Konstanten bezeichnen, die nur von absoluten Parametern abhängen.

Unterfall b: $s>2 x^{n}$. Dann gilt

$$
\begin{aligned}
& e\left(U, B_{\imath}(x)\right) \leqq C e\left(U, B_{x^{n}}(x)\right) \leqq C x_{n}{ }^{p-n} \int|D u|^{p} \\
& B_{j_{n}}^{+}\left(x^{\prime}\right) \\
& \varliminf_{(1)} C s^{p-n} \int_{B_{3,2}\left(x^{\prime}\right)}|D u|^{p} \leqq C s^{p-n} \int_{B_{2}^{+}(x)}|D u|^{p} \leqq C e\left(U, B_{2 s}(x)\right) .
\end{aligned}
$$


Fall 2: $t>x^{n}$. Aus $B_{t}(x) \subset B_{3 l}\left(x^{\prime}\right)$ folgt

$$
\begin{aligned}
& e\left(U, B_{t}(x)\right) \leqq C t^{p-n} \int_{B_{3 t}^{+}\left(x^{\prime}\right),}|D u|^{p} \leqq C s^{p-n} \int_{B_{3 s}^{+}\left(x^{\prime}\right)}|D u|^{p} \\
& \leqq C s^{p-n} \int_{B_{4 s}^{+}(x)}|D u|^{p} \leqq C e\left(U, B_{4 s}(x)\right) .
\end{aligned}
$$

Insgesamt ist damit bewiesen

$$
\text { ; } \quad e\left(U, B_{\ell}(x)\right) \leqq C e\left(U, B_{4 s}(x)\right), \quad t<s, \quad x \in \mathbf{R}_{+}{ }^{n} \cup T .
$$

Trivialerweise gilt (2) nit einer anderen Konstanten $C$, wenn rechts die Kugel $B_{s}(x)$ géwählt wird: Ist nämlich $s>t$, so hat man im Fall $s \leqq 4 t$ offenbar $e\left(U, B_{t}(x)\right)$ $\leqq C e\left(U, B_{s}(x)\right)$. Gilt $s>4 t$, so benutzt man (2) mit $s^{\prime}:=4^{-1} s$.

0

\section{Bewéis von Satz 2}

Seien $z \in B_{1 / 2}^{+}(0) \cup \dot{T}$ und $t \in(0,1 / 20)$ v́orgegeben. Es gelte

$$
\int_{B_{1}^{+}(0)}|D u|^{p} d x<\varepsilon^{p}
$$

mit einem später festzulegenden $\varepsilon>0$.

Fall $1: z^{n}>2 t$. Bezeichnet dann $\dot{a}$ den Mittelwert von $u$ iber die innere Kugel $B_{2 t}(z)$, so folgt aus (1) und der Monotonieformel

$$
f_{B_{3 t}(z)}|u-a|^{p} d x \leqq c_{1} t^{p-n} \int_{B_{z z}(z)}|D u|^{p} d x \leqq c_{2} \varepsilon^{p} .
$$

Nach : Hubini und [Mo], Theorem 3.6.1(c), existiert ein Radius $s \in[t, 2 t]$ mit $u \in H^{1, p}\left(\partial B_{s}(z), \bar{G}\right)$ und

$$
\begin{aligned}
& E\left(u, \partial B_{s}(z)\right) \leqq c_{3} t^{-1} E\left(u, B_{2 t}(z)\right), \\
& W\left(u, \partial B_{s}(z)\right) \leqq c_{3} t^{-1} W\left(u, B_{2 \ell}(z)\right)
\end{aligned}
$$

(Notationen aus dem Fortsetzungssatz). (2) und die vorstehende Rechnung ergeben

$$
E\left(u, \partial B_{s}^{\prime}(z)\right) \cdot W\left(u, \partial B_{s}(z)\right)^{\gamma} t^{p+(1-n)(y+1)} \leqq c_{4} \varepsilon^{p(y+1)}
$$

und die. Voraussetzungen des Fortsetzungssatzes sind erfüllt mit der Wahl $-\tilde{\varepsilon}:=\left(c_{4} \delta^{-\gamma-1} \varepsilon^{(\nu+1) p}\right)^{1 / x}$, vorausgesetzt man wählt $\varepsilon$ so klein, da $\beta \tilde{\varepsilon} \leqq \varepsilon_{1}$ ausfällt. Dann gibt es eine Funktion $\bar{u}^{\prime} \in H^{1, p}\left(B_{s}(z), \bar{G}\right)$ mit Randwerten $u$ und

$$
\begin{aligned}
E\left(\bar{u}, B_{s}(z)\right) & \leqq K\left\{\bar{\varepsilon} t E\left(u, \partial B_{s}(z)\right)+t^{1-p} \tilde{\varepsilon}^{-\tilde{x}} \cdot W\left(u, \partial B_{s}(z)\right)\right\} \\
& \leqq K\left\{\tilde{\varepsilon} E\left(u, B_{2 t}(z)\right)+\tilde{\varepsilon}^{-\bar{x}} t^{-p} W\left(u, B_{2 t}(z)\right)\right\}
\end{aligned}
$$

Die lokale Minimalität von $u$ impliziert $E\left(u, B_{s}(z)\right) \leqq E\left(\bar{u}, B_{s}(z)\right)$, und durch Kombination mit der vorstehenden Úngleichung folgt

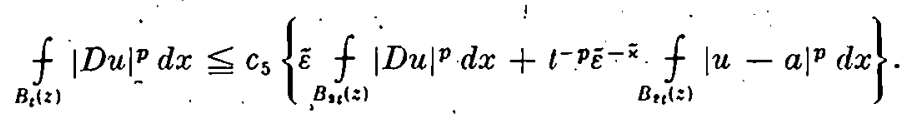


Fall 2: $0 \leqq z^{n} \leqq 2 t$. Sei jetzt $a$ der Mittelwert vón $U$ über $B_{8 t}\left(z^{\prime}\right)$, wo $z^{\prime}$ wie vorhin die Projektion von $z$ auf die Hyperebene $T$ ist. Gemä $B B_{t}(z) \subset B_{4 t}\left(z^{\prime}\right)$. ist mit $s:=4 t$

$$
\int_{B_{t}^{+}(z)}|D u|^{p} d x \leqq \int_{B_{q}\left(z^{\prime}\right)}|D U|^{p} d x
$$

Aus der Monotonieformel erhalten wir mit (1)

$$
e\left(U, B_{2 s}\left(z^{\prime}\right)\right) \leqq c_{6} e\left(U, B_{1 / 2}\left(z^{\prime}\right)\right) \leqq c_{7} E\left(u, B_{1}{ }^{-}(0)\right) \leqq c_{7} \varepsilon^{p},
$$

und wie in Fall 1 findet man einen Radius $r \in[s, 2 s]$ mit

sowie

$$
\dot{U}_{\mid \partial_{B_{r}\left(z^{\prime}\right)}} \in H^{1, p}\left(\partial B_{r}\left(z^{\prime}\right), \bar{G}\right)
$$

$$
\begin{aligned}
& E\left(U, \partial B_{r}\left(z^{\prime}\right)\right) \leqq c_{8} s^{-1} E\left(U, B_{2 s}\left(z^{\prime}\right)\right) \\
& W\left(U, \partial B_{r}\left(z^{\prime}\right)\right) \leqq c_{8} s^{-1} W\left(U, B_{2 s}\left(z^{\prime}\right)\right)
\end{aligned}
$$

Es bezeichne $E$ die affin lineare Transformation, die $B_{r}{ }^{n}\left(z^{\prime}\right)$ auf $B_{1}{ }^{n}(0)$ abbildet mit $E\left(z^{\prime}\right)=0$ und $I I$ sei die stereographische Projektion $S_{1}^{n-1} \rightarrow \mathbf{R}^{n-1}$ vom. Südpol. Man setzt

$$
\varphi(x):= \begin{cases}U(x), & x \in \partial B_{r}^{n}\left(z^{\prime}\right)^{\prime}, x^{n} \geqq 0, \\ \zeta\left(U\left(E^{-1}\left(\Pi^{-1}(E(x))\right)\right)\right), & x \in B_{r}^{n-1}\left(z^{\prime}\right),\end{cases}
$$

Offensichtlich gehört $\varphi$ zum Sobolev-Raum $H^{1, p}\left(\partial B_{r}^{+}\left(z^{\prime}\right), \bar{G}\right)$ mit Bild $\left(\varphi_{\mid T}\right) \subset S$. Mit einfacher Transformation impliziert (4)

$$
\begin{aligned}
& s^{p+(1-n)(y+1)} E\left(\varphi, \partial B_{r}^{+}\left(z^{\prime}\right)\right) \cdot W\left(\varphi, \partial B_{r}^{+}\left(z^{\prime}\right)\right)^{\gamma} \\
& \leqq c_{9} e\left(U, B_{2 s}\left(z^{\prime}\right)\right)\left(\underset{B_{z s}\left(z^{\prime}\right)}{f}|U-a|^{p} d x\right)^{\gamma} \\
& \leqq c_{10} e\left(U, B_{2 s}\left(z^{\prime}\right)\right)^{1+\gamma} \leqq c_{z} c_{10} \varepsilon^{p(y+1)}=c_{11} \varepsilon^{p(y+1)} .
\end{aligned}
$$

Da die Vollkugel Bi-Lipschitz äquivalent ist zur Halbkugel, kann man den Fortsetzungssatz anwenden, vorausgesetzt $c_{11} \varepsilon^{(1+\gamma) p} \leqq \tilde{\varepsilon}^{x} \delta^{y+1}$, und erhält eine Fortsetzung $\Phi$ von $\varphi$ mit

$$
\therefore \quad E\left(u, B_{r}^{+}\left(z^{\prime}\right)\right)^{\prime} \leqq E\left(\Phi, B_{r}^{+}\left(z^{\prime}\right)\right) \leqq K\left(\tilde{\varepsilon} E\left(U, B_{2 s}\left(z^{\prime}\right)\right)+s^{-p} \tilde{\varepsilon}^{-\tilde{x}} W\left(U, B_{2 s}\right)\right\} .
$$

Schließlich benutzt $\operatorname{man} B_{2 s}\left(z^{\prime}\right) \subset B_{10 t}(z)$ sowie :

$$
\because \int_{B_{z y}\left(z^{\prime}\right)}|U-a|^{p} d x \leqq c_{12} \int_{B_{10}(z)}\left|U-(U)_{10 t}\right|^{p} d x
$$

was letztendlich auf die Abschätzung

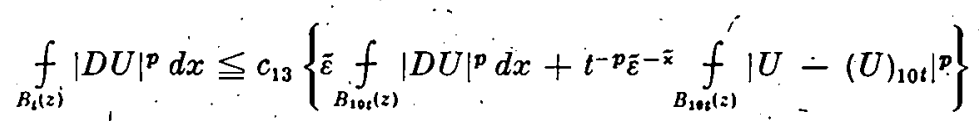

führt. Vergleicht man dieses Ergebnis mit (3), so sieht man unmittelbar, da B (5) für alle Punkte $z \in B_{1 / 2}(0)$ und Radien $0<t<1 / 20$ richtig ist. Mit einer Variante von $[G], ' V$, Prop. 1.1, folgt sofort die, Behauptung, wenn man $\varepsilon$ und damit $\bar{\varepsilon}$ genïgend klein wählt. 


\section{Beweis von Satz 1}

Für kleine Radien $R$ kann man $E\left(u, B_{R}{ }^{+}(0)\right)<\varepsilon^{n}$ mit noch zu bestimmendem $\varepsilon$ erreichen. Sei $d:=$ dist $(\cdot, S)$ die Abstandsfunbtion zu $S$ gemessen in der Mannigfaltigkeit $Y . d$ ist Lipschitz, also gehört $v:=d \circ U z u H_{\operatorname{loc}}^{1, n}\left(\mathbf{R}^{n}\right)$ mit $v_{\mid T}=0$. Für $z \in B_{R / 2}^{+}(0) \cup T$. und $0<t<R / 20$ unterscheiden wir wie vorhin die folgenden Fälle:

Fall $1: z^{n}>2 t$. Dieselben Rechnungen wie im Beweis von Satz 2 führen auf Ungleichung (3), wenn man $F\left(u, B_{s}(z)\right) \leqq F\left(\bar{u}, B_{s}(z)\right)$ beachtet und die Elliptizität des Funktionals $F$. ausnutzt:

Fall $2: 0^{\prime} \leqq z^{n} \leqq 2 t$. Jetzt wählt man den Radius $r \in[s, 2 s]$ so, daß neben (4) auch noch die Bedingung osc $v<$ const $\cdot \varepsilon$ erfüllt ist, was nach einer Variante des $\partial_{\underline{B}}\left(z^{\prime}\right)-$

Courant-Lebesgue Lemmas (vgl. [J2], Lemma 1) möglich ist. Für genügend kleine Werte von. $\varepsilon$ folgt sodann, daß $U_{\mid \partial_{r}\left(z^{\prime}\right)}$ Bild in einer geeigneten Tubenumgebung von $S$ hat, auf der die Nächste-Punkt-Projektion $\zeta$ erklärt ist. Dies liefert (5) mit denselben Arguménten wie oben.

\section{LITERATUR}

[B] BaLdes, A.: Harmonic mappings with partially. free boundary. Manus. math. 40 (1982), 255-275.

[D] 'DuZaAR, F.: Variational inequalities and harmonic maps. J. Reine Angew. Math. 374 (1987), 39-60.

[D, F 1] DozaAR, F., and M. Fuchs: Variational problems with non-convex obstacles and an integral constraint. Math. Z. 191 (1986), 585-591.

[D, F2] DuzaAk, F., and M. Focus: Optimal regularity theorems for variational problems with obstacles. Manus. math. 56 (1986), $209-234$.

[F1] Fodus, M.: Variational inequalities for vector-valued functions with non-convex obstacles. Analysis 5 (1985), 223-238. .

[F2] Fucus, M.: Some remarks on the boundary regularity for minima of variational problems with obstacles. Manus. math. 54 (1985), 107-119.

[F3] Fuchs, M.: A note on removable sigularities for certain vector-valued obstacle .problems. Arch. Math. 48 (1987), 521-525.

- [F4] Fuchs, M.: A regularity theorem for energy minimizing maps of Riemanniań manifolds. Comm. Part. Diff. Equ. 12 (1987), 1309-1321.

[F5] Fuchs, M.: An elementary partial regularity proof for vector-valued obstacle problems. Math. Ann. 279 (1987), 217-226..

[F6] Fuchs, M.: p-harmonische Hindernisprobleme. Habilitationsschrift. Univ. Düsseldorf 1987 .

[F, H] Fusco, N.; and J. Hutchisson: Partial regularity for minimizers of certain functionals having non-quadratic growth. Ann. Math. Pura Appl. (erscheint).

[G] Gaquinta, MI.: Multiple integrals in the calculus of variations and nonlinear elliptic systems. Princeton: University. Press 1983.

[Go] GorNık, K.: Ein Stetigkeitssatz für Variationsprobleme mit Ungleichungen als Nebenbedingungen. Math. Z. 152 (1976), 89-97.

[G, H, N] Grüteh, M., Hildebrandt, S., and J. C. C. Nitsche: Regularity for stationary surfaces of constant mean curvature with free boundarics. Acta Math. $156(1986)$, $119-152$.

[G, J] . Golliver, R., and J. Jost : Harmonic maps which solve a free boundary problem. Preprint 796. SFB 72, Univ. Bonn 1986.

[J1] Jost, J.: On the regularity of minimal surfaces with free boundaries in Riemannian manifolds. Manus. miath. 56 (1986), 279-291. 
[J 2] Jost, J.: A conformally invariant variational problem for mappings between Riemannian manifolds. Preprint 1985.

[Mo] Morrey, C. B. jr.: Multiple integrals in the calculus of variations. Berlin-Heidelberg-New York: Springer-Verlag 1968.

Manuskripteingang: 24. 07. 1987

VERFASSER:

Dr. Martin Fuchs

Mathematisches Institut der Universität

Universitätsstr. 1

D -4000 Düsseldorf 